\title{
Sistemas de inocuidad y calidad de tres microempresas agroindustriales de San Carlos, Costa Rica
}

\author{
Carmen Andrés Jiménez \\ Universidad Estatal a Distancia (UNED), 474-2050 Mercedes de Montes de Oca, San José-Costa Rica; candres@uned.ac.cr
}

Recibido 11-VIII-2010 Corregido 1-X-2010 Aceptado 10-XI-2010

\begin{abstract}
Innocuity and quality systems in three small agro-industrial companies from San Carlos, Costa Rica. Agroindustry contributes to the economical well being of a country because it helps small producers to leave the raw materials model and to add value to their products by selling finished goods to the national and international market. However, without good manufacturing practices and quality controls, agroindustry cannot develop correctly. There is a series of rules which need to be followed by all employees at all stages of the process in order to achieve high quality. This investigation gathered information related to the innocuity and quality of a snack factory, a noni fruit juice factory and a milk caramel factory in La Perla, San Carlos, Costa Rica, through surveys and checklists. There were differences among the companies: the snack factory complies with more aspects related to innocuity and quality, the noni factory fulfills some requirements but can improve the quality of its products and the milk caramel factory has not started implementing any innocuity and quality programs. I present recommendations for all companies and hope this article will help other small companies in developing countries.
\end{abstract}

\section{KEY WORDS}

Good Manufacturing Practices, finished goods, quality, innocuity, nutrition, small industries.

\begin{abstract}
RESUMEN
La agroindustria contribuye al desarrollo económico de un país ya que ayuda a pequeños productores a transformar sus materias primas en productos de valor agregado así como a exportarlos y ofrecerle al consumidor opciones nutritivas. Pero, sin buenas prácticas de manufactura y controles de calidad, la agroindustria no puede funcionar de manera correcta. Existen una serie de reglas que necesitan ser cumplidas por todos los empleados en todas las etapas del proceso para lograr productos de alta calidad. Esta investigación recopiló y analizó información relacionada con la inocuidad y calidad de una tostadora, una microempresa de noni y una microempresa de cajetas en La Perla, a través de una encuesta, una lista de chequeo y una prueba de diferencias entre proporciones. Se encontraron diferencias significativas entre las microempresas. La tostadora es la que cumple más aspectos básicos de inocuidad y calidad, la microempresa de noni cumple con algunos de los requerimientos para mejorar la calidad de sus productos y la microempresa de cajetas no ha empezado a implementar aún estos programas. Un sistema básico de inocuidad y calidad debe ser implementado en todas las microempresas.
\end{abstract}

\section{PALABRAS CLAVE}

Buenas prácticas de manufactura, productos terminados, calidad, inocuidad, nutrición, microempresas.
La agroindustria contribuye de forma significativa al desarrollo económico de un país subdesarrollado debido al valor agregado y nutricional que los productores obtienen a través de ella, lo cual genera mayores ingresos. Además, los productos agroindustriales representan la mayor parte del sector manufacturero y suelen ser la principal fuente de exportación (Austin 1981).

Para que la calidad e inocuidad de los productos elaborados en una agroindustria sea la óptima, es necesario cumplir con una serie de requisitos: buenas prácticas de manufactura y programas de soporte (ambos relacionados con la inocuidad) y sistemas de gestión de calidad. Estas exigencias se establecen en el Reglamento técnico centroamericano (MINECO et al. 2007), en la norma ISO 22 000: 2005 (Færgemand 2008, ISO 2009), las normas ISO 9001, relacionadas con Sistemas de Gestión de calidad (INTECO 2010).

La implementación de los estándares antes mencionados demanda recursos económicos, humanos y técnicos que son difíciles de accesar por parte de las microempresas agroindustriales de Costa Rica. 
Lo anterior se ha ido agravando debido a la crisis económica mundial, ya que el comportamiento del PIB del primer trimestre del 2009 en el sector industrial manufacturero de Costa Rica, dentro del cual se encuentra la agroindustria, fue de $-14.8 \%$, mientras que en el año 2008 fue de -4.2 puntos porcentuales y en el año 2007 de 7.0 puntos porcentuales (Programa Estado de la Nación 2009).

Sin embargo, a partir de febrero del 2010, el Sistema de Banca para el desarrollo prestó una cantidad considerable a las pequeñas y medianas empresas (PYMES) de Costa Rica y las estadísticas mostraron que el $94 \%$ de las personas que recibieron dinero fueron de microempresas (Mayorga 2010). De la mano con lo anterior, el Banco de América Central de Costa Rica (Grupo BAC Credomatic) desarrolló un programa con las PYMES para que puedan utilizar en forma más eficiente la energía, financiando sustitución de equipos de iluminación, motores y plantas (Leitón 2010).

Además del apoyo económico y ambiental que las entidades públicas y privadas están ofreciendo, la UNED ha estado trabajando para ayudar a las microempresas de la zona en diferentes aspectos mediante capacitaciones, consultoría, investigaciones y motivación. Todo esto se ha logrado mediante el CITTED (Centro de Investigación, Transferencia Tecnológica y Educación), el cual fue fundado en el año 2005, para ayudar en diferentes actividades económicas de la zona.

Los ejes transversales bajo los cuales trabaja el CITTED son los siguientes: tecnologías de la información y la comunicación (TIC), socioeducación, sistemas productivos, medio ambiente y eco turismo y biodiversidad (Murillo 2009).

Los ejes en los que se basó la primera fase del presente proyecto fueron la socioeducación y los sistemas productivos. Estos ejes contemplan el hecho de que mediante la educación de la población en inocuidad y calidad, se construye la base necesaria para pasar a la siguiente fase que los llevaría a montar un sistema productivo. Las costumbres, cultura y relaciones sociales conforman la vida campesina en este lugar y las acciones por parte del CITTED deben basarse en el contexto histórico de estas personas porque el mismo determina su perspectiva y su visión ante los retos (Cornejo 2009).

El objetivo principal de este proyecto de investigación consistió a recolectar la información suficiente de tres microempresas interesadas en mejorar la elaboración de sus productos, analizarlos y generar un plan de acción que mita la implementación de los aspectos más urgentes a mejorar.

Las microempresas seleccionadas fueron las siguientes: una tostadora de maíz, plátano y malanga; una microempresa de jugo de noni y una microempresa de cajetas de coco. Se escogieron debido a su interés por mejorar la calidad de sus productos y por su capacidad de trabajo. Además, se capacitó a los involucrados en estas microempresas, trazando una base de conocimiento para poder trabajar y mejorar en las áreas de inocuidad y calidad.

\section{METODOLOGÍA}

\section{Procedimiento}

Se realizó una gira al campo en donde se evaluó el estado actual del proceso con el que tres microempresas están elaborando sus productos alimenticios. Se analizó la información para identificar fortalezas y debilidades y preparar un plan de mejora.

\section{Muestra utilizada}

Se visitaron tres microempresas de la zona de La Perla, San Carlos, productoras de alimentos, que se encuentran en una etapa incipiente en lo que respecta a los sistemas de inocuidad y calidad.

La primera microempresa es una Tostadora de productos fritos elaborados a partir de plátano, yuca, malanga. Tiene un total de 10 empleados y sus clientes son pulperías y supermercados de la zona.

La segunda es una microempresa jugo de noni. Esta empresa la opera la misma dueña (una empleada) y sus clientes son intermediarios que le compran su jugo para reempacarlo y venderlo a San José.

La tercera es una microempresa de cajetas, en la cual hay 2 empleados. Sus clientes son pulperías de la zona.

\section{Instrumentos de investigación}

Esta evaluación se realizó mediante la aplicación de dos herramientas cualitativas: una entrevista y listas de chequeo. La entrevista se aplicó una sola vez y se incluyeron temas relacionados con la producción, el control de calidad y la seguridad alimentaria (ver Apéndice 1).

Las listas de chequeo aplicadas también una vez incluyeron todos los rubros relacionados con buenas prácticas de manufactura, control de calidad, producción, salud ocupacional y medio ambiente (ver Apéndice 2).

\section{Diseño experimental}

Una vez que se aplicaron las herramientas, se procedió a analizar los datos con el objetivo de determinar el porcentaje de cumplimiento de cada microempresa con respecto a la inocuidad y calidad. 
Se utilizó una prueba de diferencia entre proporciones para determinar si existen diferencias significativas entre las microempresas, tanto a través de la encuesta realizada como a través de la lista de chequeo.

\section{RESULTADOS}

\section{Estado actual de las microempresas}

Se le aplicó una entrevista a cada microempresa para determinar el cumplimiento general con respecto a los sistemas de gestión de inocuidad y calidad y de forma general se encontraron deficiencias que deben ser corregidas a corto plazo, de lo contrario los productos que elaboran pueden sufrir las consecuencias, así como la buena fama las microempresas. Los resultados de esta encuesta pueden verse en la Fig. 1.

Como siguiente paso para realizar el diagnóstico de las tres microempresas, se aplicó la lista de chequeo de inocuidad y calidad, cuyos resultados para cada empresa se muestran en la Fig. 2.

Al $1 \%$ de confianza, aplicando la prueba de diferencia entre proporciones, se determinó que existe diferencia significativa entre las tres microempresas.

\section{DISCUSIÓN}

La evaluación de la inocuidad y calidad de las empresas fue realizada en dos etapas: a) Aplicación de una encuesta a cada gerente de la microempresa; b) Aplicación de las listas de chequeo a cada microempresa para evaluar la inocuidad y calidad de sus productos.

De manera general se encontró que las empresas no cuentan con documentación que respalde los procesos relacionados con los sistemas de gestión (ni documentos escritos ni registros). Además, no se cumple con los lineamientos establecidos para la infraestructura, controles y prevenciones que una agroindustria debe tener.

Si bien es cierto, la falta de recursos hace que las microempresas diagnosticadas no tengan todos los requisitos mínimos para evitar los riesgos sanitarios o de calidad; la higiene y dedicación con la que trabajan hacen que su producto sea de alta calidad. Sin embargo, es necesaria la asesoría profesional, para que puedan proteger su empresa de posibles demandas o problemas con clientes. La FAO (2010) ha establecido que la calidad de los alimentos es un pre requisito para el éxito de cualquier empresa en un mercado agrícola cada vez más competitivo, la cual se está convirtiendo cada vez más en una ventaja para las microempresas que lo adoptan.

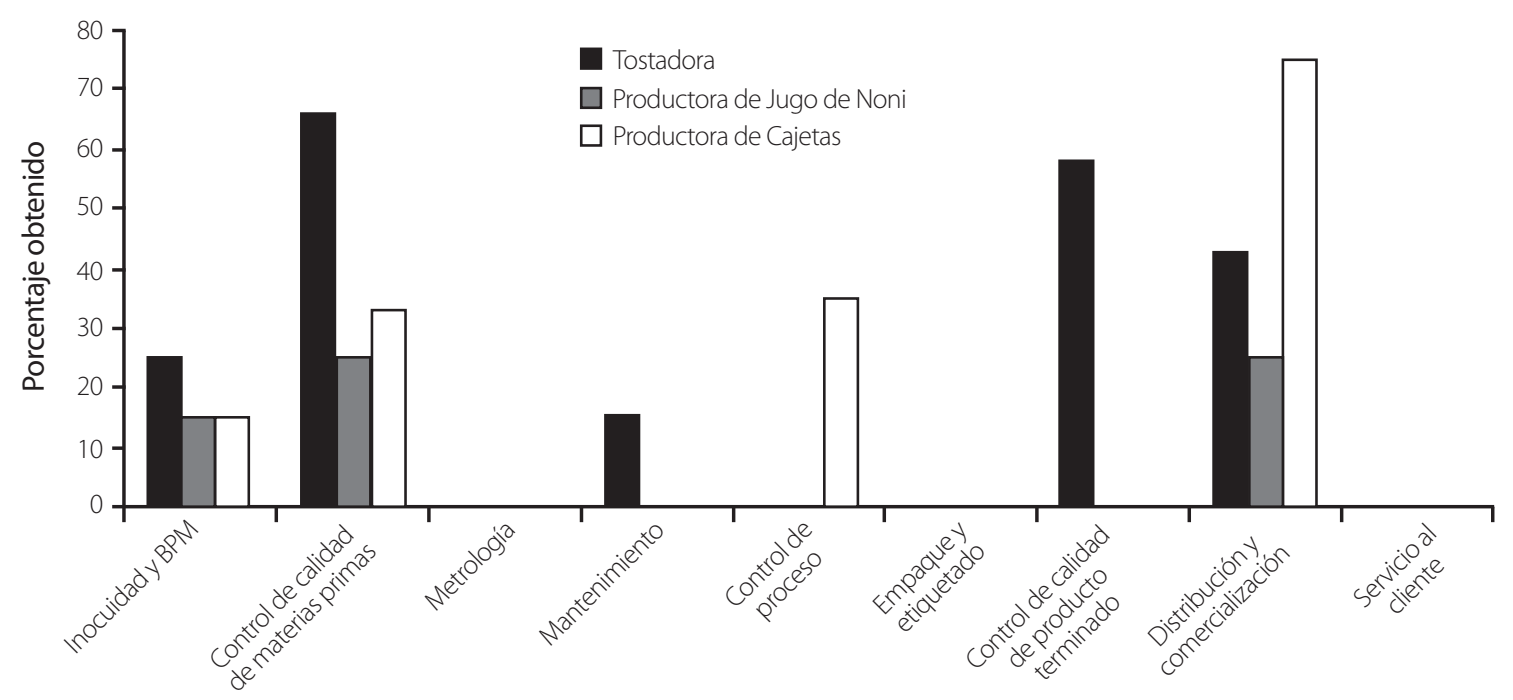

Aspectos evaluados

FIG. 1. Cumplimiento en porcentajes de cada aspecto de la encuesta realizada a cada microempresa en relación a la gestión de la inocuidad y calidad. Los aspectos de metrología, etiquetado y empaquetado y servicio al cliente se evaluaron pero hubo un $0 \%$ de cumplimiento. 


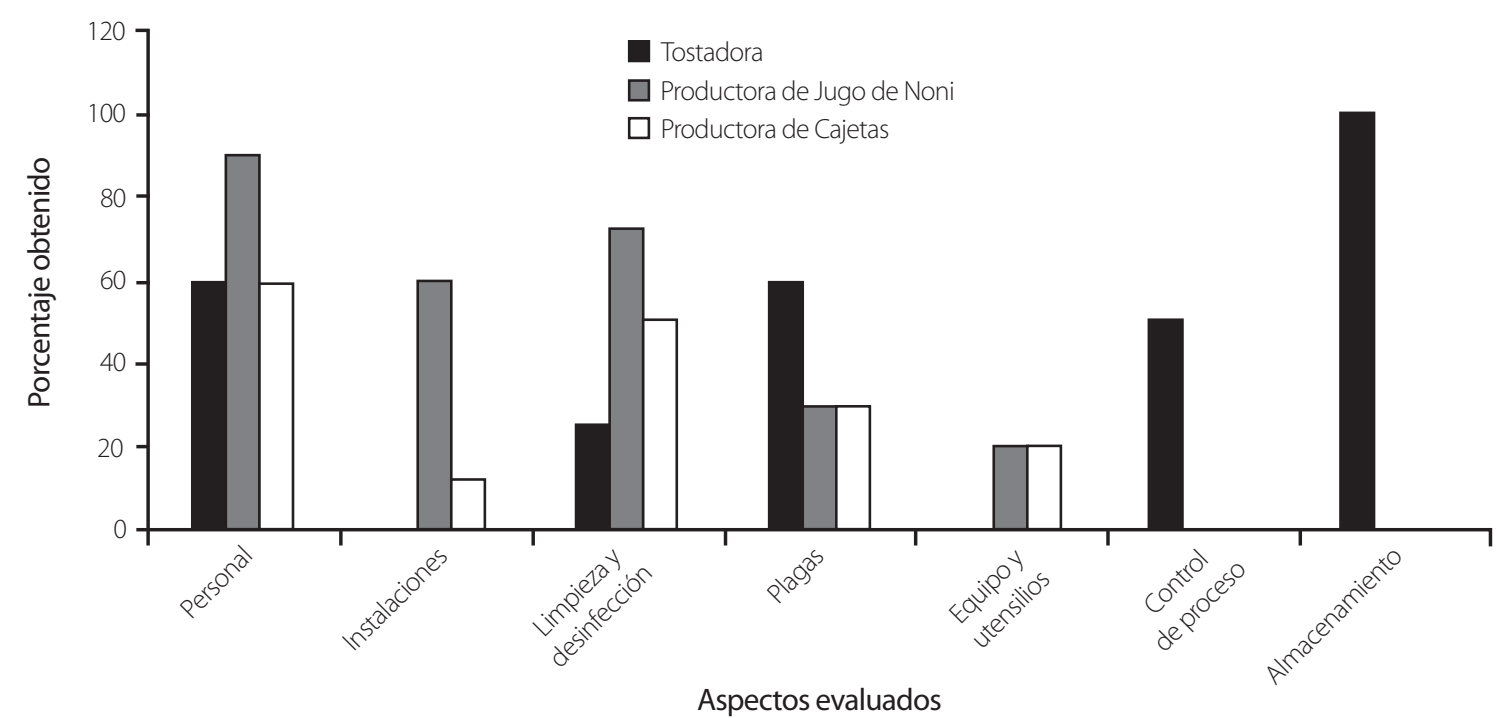

FIG. 2. Calificación final que cada microempresa evaluada obtuvo después de la aplicación de la lista de chequeo en relación a la gestión de la inocuidad y calidad.

Debido a que cada herramienta utilizada tiene aspectos relacionados entre sí, los mismos se analizarán a continuación, tomando en cuenta los resultados obtenidos en ambas herramientas.

\section{Buenas prácticas de manufactura}

En la Fig. 1, se puede observar que la Tostadora cumple en un $25 \%$ y las otras dos microempresas cumplen apenas en un $15 \%$ de los aspectos evaluados. La Tostadora y la microempresa de cajetas cumplen con los lineamientos para limpieza y desinfección y la microempresa de noni realiza control de la inocuidad del agua. Sin embargo, ninguna de las microempresas cumple con el resto de los aspectos de este punto.

Un mal manejo de los alimentos es uno de los factores que preocupan la salud pública de las pequeñas y medianas industrias (Bostwana 2005). Y el hecho de que dos de las microempresas evaluadas no realicen un control de la inocuidad del agua implica un riesgo muy grande para la salud de los consumidores y para la fama de las microempresas. La norma ISO 22000 (ISO 2009) estipula que el agua utilizada como ingrediente de alimentos, debe cumplir con requerimientos específicos de calidad y microbiología.

Para evaluar el aspecto de Buenas Prácticas de Manufactura (BPM) con la lista de chequeo, se realizaron observaciones relacionadas con el aseo personal, la higiene de las instalaciones y la aplicación de BPM. Todas las microempresas deben mejorar, lo cual se corroboró observando que solamente la Tostadora solicita a su personal utilizar uniforme, pero ninguna de las tres microempresas analizadas tiene una estación de lavamanos al ingreso a la planta. De acuerdo al Reglamento Centroamericano de Buenas Prácticas, se debe exigir a los operarios que se laven con agua, jabón y desinfectante en diferentes momentos del día y si no existe una estación de lavado a la entrada de la zona de proceso, la probabilidad de que se laven las manos es muy baja (Comex 2010).

Esta recomendación es factible para las microempresas y no implica una inversión grande de dinero, por lo que es probable que la implementen en un plazo relativamente corto.

\section{Limpieza y desinfección}

Con respecto a estos procedimientos, la normativa exige que haya un procedimiento estructurado de control y orden (ISO 2009). Este aspecto fue evaluado también al aplicar la lista de chequeo y no se encontraron registros en donde se pueda evidenciar la realización de estos procesos. Esto es importante que la empresa lo implemente.

A pesar de realizar la limpieza de forma adecuada en las tres microempresas, la documentación detrás de un sistema SSOP (Sanitization Standard operacional procedures; 
en español: procedimientos estándares de operación de limpieza), es deficiente y según la normativa es necesaria como respaldo para la microempresa en caso de una auditoría o inspección por parte del gobierno y para controlar debidamente estos procesos (R. López, com. pers.).

Las empresas deben escribir procedimientos de limpieza y desinfección, así como empezar a registrar estas operaciones y controlarlas. La escritura de este y el resto de los procedimientos, aunque no implica una inversión económica, no es tan factible para las microempresas sin la ayuda de un profesional en el área de Agroindustria que pueda definir los lineamientos. En este caso la UNED seguirá dando asesoría a las microempresas dentro de lo que sea posible.

\section{Control de plagas}

La norma establece que debe haber un programa escrito de control de plagas, condiciones especiales de infraestructura, los químicos utilizados para las fumigaciones deben almacenarse de manera que no contaminen el alimento, debe haber un proceso de erradicación de pestes y todo el programa debe ser monitoreado (ISO 2009).

Este aspecto fue evaluado también con la lista de chequeo y se encontró que no se cumple ninguno de los requisitos anteriores. Sin embargo las tres microempresas realizan sus fumigaciones al menos una vez por mes y la planta de producción se ve libre de plagas. La Tostadora además, tiene trampas de ratones aseguradas al piso y los productos para fumigar están almacenados de manera que no contaminen.

Para mejorar en este aspecto es necesaria una inversión inicial, por lo que la implementación de la misma se podrá hacer parcialmente, con la ayuda de la UNED en asesoría profesional y si las microempresas deciden invertir.

\section{Control de calidad de materias primas}

Todas las microempresas cumplen con el sistema FIFO (first in-first out, primero que entra primero que sale), el cual debe ser verificado periódicamente según la norma, acción que las empresas sí no están cumpliendo.

Con respecto a la calidad de las materias primas que se utilizan, solamente la tostadora cuenta con proveedores certificados en sistemas de calidad. Sin embargo, no recibe certificados de análisis en cada lote de producción, lo cual es muy importante que se implemente.

Ninguna de las microempresas tiene un procedimiento estandarizado para el control de la calidad de las materias primas, el cual consiste en seleccionar y gestionar los mejores proveedores para cada una de las materias primas utilizadas mediante inspecciones y envío de requerimientos, además de un monitoreo periódico y un chequeo constante de los materiales que se reciben, lo cual abarca el chequeo del camión, análisis documentado de cumplimiento de requerimientos (ISO 2009).

El no contar con un sistema de numeración de lotes, de registro del FIFO ni de chequeo constante de la bodega. Lo anterior es muy importante porque facilita los procesos de administración del producto comercializado, agiliza los procesos de trazabilidad desde las materias primas hasta el producto terminado y hace posible que los productos de la bodega estén siempre frescos y no se deterioren (Alvear 2010).

La implementación de este aspecto es más sencilla y con ayuda de la UNED, es probable que las microempresas la puedan realizar.

\section{Metrología}

La metrología o confirmación metrológica, para la cual no cumple ninguna de las microempresas evaluadas. Esta evaluación incluye calibración o verificación de los equipos utilizados para elaborar y analizar los alimentos (ISO 2009).

Si consideramos el ejemplo de la empresa que medía la temperatura de pasteurización de su producto todos los días y siempre le daba bien $\left(85^{\circ} \mathrm{C}\right)$ y que a pesar de eso seguía perdiendo lotes enteros de producto hasta que se dieron cuenta de que el termómetro no estaba bien calibrado y en realidad la temperatura del producto no estaba llegando ni a $60^{\circ} \mathrm{C}$; podemos entender que la calibración de los equipos es clave para la calidad del producto final (E. Mora com. pers.).

Este tema requiere de una inversión inicial importante, además de que el mantenimiento también requiere de gastos por parte de la empresa. No es tan factible que las microempresas lo implementen, pero se pueden establecer prioridades dentro de las cuales las empresas decidan invertir para no comprometer la calidad de los productos.

\section{Mantenimiento preventivo}

El siguiente aspecto, mantenimiento preventivo, es un aspecto en el cual solamente la Tostadora ha trabajado un poco, en donde existe un procedimiento general de mantenimiento preventivo, aunque no está completo.

El mantenimiento preventivo incluye todo el equipo e infraestructura que tiene algún contacto con el producto, materias primas, materiales de empaque o etiquetado. Es necesario que exista un plan de trabajo en el que se 
tengan definidos todos los trabajos a realizar por mes que permitan un correcto funcionamiento de los equipos y se pueda prevenir las fallas antes de que generen una contaminación en los alimentos.

En la inspección realizada con la lista de chequeo se encontró que la Tostadora ha tomado medidas para evitar que entren plagas a la planta de producción, además de tener los alrededores limpios, desagües con rejilla, paredes limpias y cedazo en las ventanas. Estos aspectos no se encontraron en las otras dos microempresas.

Además de lo anterior, el no tener rendijas, baños y lockers en buen estado, uniones redondeadas, protectores en lámparas; entre otros, es un problema importante para las microempresas ya que el cumplimiento de estos rubros previene problemas de inocuidad y calidad.

La implementación de este aspecto se puede iniciar de manera que se planee lo que se debe hacer mes a mes, priorizando lo que sea factible económicamente para la microempresa.

\section{Control de proceso}

El control de proceso es importante para que todos los parámetros bajo los cuales están formulados los productos de las microempresas estén siempre bajo los límites establecidos. En otros estudios se han utilizado diferentes herramientas mediante las cuales se pueden controlar y mejorar los procesos, entre las cuales se encuentran: gráficas dinámicas y de control, paretos, diagramas de causa-efecto, diagramas de dispersión (Evans \& Lindsay 2005).

El control de proceso se evaluó tanto a través de la encuesta como a través de las listas de chequeo y se encontró que solamente la Tostadora realiza algún control, pero es mínimo (no existe documentación que lo respalde y los controles no son constantes). Este problema puede tener implicaciones en la calidad del producto final y la cantidad de producto no conforme aumenta considerablemente.

Este aspecto es factible de controlar con ayuda de la UNED, se puede guiar al personal para que lo implemente y lo siga aplicando.

\section{Empaque y etiquetado}

Ninguna de las microempresas tienen sistemas que lo respalden implementados. En este rubro, debe existir un procedimiento mediante el cual se describa cómo se desarrollarán los empaques y etiquetas de cada producto a lanzar al mercado, cómo se harán los cambios de empaque y etiquetas para mejorar la imagen del producto y cómo se seguirán las normas del MEIC (en el caso de Costa Rica) para este rubro.

En un estudio realizado en Venezuela con 13 micro, pequeñas y medianas empresas, se encontró que el $7.7 \%$ tenía el registro sanitario (Ablan et al. 2007). De las microempresas evaluadas, solamente la Tostadora cumple con este criterio. Sin embargo, es un aspecto muy importante que debe promoverse.

\section{Control de producto terminado}

Se encontró que solamente la Tostadora es la que cumple con este aspecto en el sentido de tener un sistema FIFO y tener un sistema definido para el manejo de producto no conforme, producto en cuarentena y producto de desecho. En un estudio realizado en México con 27 PYMES, se encontró que la calidad de los productos y el control de proceso son aspectos que no cumplen con las especificaciones de la norma (Mateos et al 2009).

El destino final que un producto no conforme pueda tener depende de resultados de análisis microbiológicos, físico químicos o sensoriales. Esto debido a que un producto no conforme puede estar retenido debido a un problema en el sabor (por no tener un ingrediente), un problema en la apariencia (por etiquetas equivocadas o una tapa de color diferente o un empaque en mal estado) o un problema en su inocuidad (por contaminación microbiológica).

Muchas veces el producto no conforme puede reprocesarse (es decir, utilizar pequeñas cantidades del producto defectuoso en producto nuevo para aprovecharlo). Es necesario que el producto sea almacenado de manera que su inocuidad, calidad y trazabilidad no se vean comprometidas (ISO 2009).

De acuerdo al Reglamento Centroamericano de Buenas Prácticas de Manufactura, es exigido que toda planta cuente con la documentación necesaria que permita la verificación de la ejecución de sus procesos, incluidos los de manejo de producto no conforme (Comex 2010).

Para la implementación de este aspecto, se necesita de la asesoría de la UNED para que se pueda iniciar de una manera correcta y se le pueda dar el seguimiento correcto. Para este y el resto de los temas planteados a las microempresas, la aprobación de futuros proyectos de investigación y extensión de la Cátedra serán claves para el éxito de los mismos.

\section{Distribución y comercialización}

Todas las microempresas cuentan con sistemas que regulen estos procesos debido a que necesitan vender sus 
productos. Todas tienen definidos los canales de distribución y a cuál mercado van dirigidas. Sin embargo los cuidados a tomar en la cadena de distribución (protección del producto y manejo del mismo en los puntos de venta), no es un punto que se esté cuidando.

\section{Servicio al cliente}

Este es un tema de vital importancia en las normas ISO y en ninguna de las empresas se ha implementado como se debería. Según la normativa ISO 9001:2008, las organizaciones dependen de sus clientes por lo que deben comprender sus necesidades, satisfacer sus requisitos y esforzarse en exceder las expectativas de los mismos (INTECO 2006).

Es indispensable que las microempresas implementen un procedimiento mediante el cual describan cómo se atenderán las quejas de los clientes, cómo se realizará el "recall" de producto (retiro de producto del mercado cuando tiene algún defecto) y las políticas de la microempresa en torno a estos temas (ISO 2009).

De igual forma que los aspectos anteriores, las microempresas necesitan dirección profesional para poderlos implementar y su posibilidad es buscar ayuda de la UNED.

\section{Documentación}

Con respecto a la documentación, en ninguna de las microempresas existen registros que respalden todo el trabajo ni se realizan análisis físico químicos o microbiológicos que respalden la aplicación de las buenas prácticas de manufactura (BPM). Sin embargo, la FAO estipula que la inocuidad es el primer paso a seguir, sin ella no sirven de nada la documentación y los sistemas de gestión de calidad (FAO 2010).

Lo anterior estipula una jerarquía de acción que no significa de ninguna manera que no se puede iniciar la escritura de procedimientos y registros en las microempresas mientras se inicia la capacitación y la concientización de todos los empleados.

Se aplicó la prueba de diferencia entre proporciones y al $1 \%$ de confianza, se encontró que existe diferencia significativa entre los resultados obtenidos a través de la encuesta y la lista de chequeo para cada una de las empresas.

De acuerdo a Huss (1994), se deben definir los objetivos con los que se va a trabajar año con año, y se debe corroborar que las políticas que se vayan implementando sean seguidas por el personal. Es por eso que se elaboró un plan de mejora que se encuentra en los Apéndices 3 y 4.

Para poder implementar los aspectos antes mencionados, es muy importante que la gerencia de cada empresa tome conciencia de la importancia de los mismos. Además, en los Apéndices 3 y 4 se recomienda la elaboración de manuales que abarquen todos los temas prioritarios para poder controlar los aspectos mencionados con registros de una manera periódica y se puedan seguir haciendo investigaciones en el futuro.

\section{CONCLUSIONES}

A partir de la evaluación realizada sobre la inocuidad y calidad de las tres microempresas en La Perla, se encontró que ninguna tiene documentación que respalde las acciones que están realizando.

Tampoco se encontraron registros que corroboren los aspectos evaluados, situación que es importante solucionar para tener un respaldo en caso de una auditoría ya sea por parte del Ministerio de Salud o un cliente.

Los aspectos relacionados con el sistema de gestión de la inocuidad tales como buenas prácticas de manufactura, mantenimiento preventivo, limpieza y desinfección y control de plagas, no se cumplen por parte de ninguna de las microempresas como se esperaba, por lo que es urgente empezar a trabajar en los mismos.

Los rubros relacionados con los sistemas de gestión de calidad tales como metrología, control de materias primas y producto terminado, control de proceso, servicio al cliente y mantenimiento preventivo, deben trabajarse fuertemente en las tres microempresas debido a que no se cumple con la mayoría de ellos.

Los aspectos relacionados con distribución y comercialización son más respetados por las empresas porque han iniciado en ese sentido para poder obtener ingresos. Sin embargo pueden mejorarse.

\section{AGRADECIMIENTOS}

Agradezco a todo el personal administrativo y técnico del CITTED por todo el esfuerzo realizado y el apoyo tan especial que me han dado en todo este proceso, en especial Allan, Daniel y Elvis. También a Annays Benavides, a Enilda Fernández y a Mayda Benavides por atendernos en sus microempresas y por el interés que pusieron en nuestras recomendaciones.

\section{REFERENCIAS}

Ablan, E., A. Medina \& M. Sánchez. 2007. Una ventana hacia la innovación: etiquetado nutricional en la producción de las micro y PYMES alimentarias en tres municipios del estado 
Mérida, Venezuela. Agroalimentaria 25:85-93 (http:// www.saber.ula.ve/bitstream/123456789/17943/1/articulo5.pdf; consultado 30 de Setiembre, 2010).

Alvear, J. 2010. Plan de implementación de un sistema de trazabilidad para productos obtenidos a partir de palmito en una empresa agroindustrial y comercialización en el mercado nacional. (http://bibdigital.epn.edu.ec/handle/15000/2101; consultado 7 de Agosto, 2010).

Austin, J. 1981. Agro industrial project analysis. Johns Hopkins University, Baltimore, Maryland, EEUU.

Bostwana. 2005. Assuring food safety and quality in small and medium size food enterprizes. (http://webcache.googleusercontent.com/custom?q=cache:2y5R_k3KKJkJ:ftp:// ftp.fao.org/docrep/fao/meeting/010/j6022e.pdf+GMP,+ food+industries $\& \mathrm{~cd}=2 \& \mathrm{hl}=\mathrm{en} \& \mathrm{ct}=\mathrm{clnk} \& \mathrm{client}=$ googlecoop-np; consultado 7 de Agosto, 2010).

Comex. 2010. Reglamento centroamericano de buenas prácticas de manufactura de la industria de alimentos y bebidas. (http://www.google.co.cr/search?hl=es\&source=hp\&q=r eglamento+centroamericano+de+buenas+practicas $+d$ e+manufactura\&rlz=1R2GGLR_esCR332\&aq =1\&aqi=g3\& $\mathrm{aql}=\& o q=$ Reglamento+centroamericano+de+\&gs_rfai=; consultado 7 de Agosto, 2010).

Cornejo, E. 2009. Gira de investigación al CITTED en La Perla de San Carlos. Exposición sobre aspectos sociológicos del CITTED. La Perla, San Carlos, Costa Rica.

Evans, J.R. \&W. Lindsay. 2005. Administración y control de la calidad. (http://scholar.google.co.cr/scholar?q=Control+de+ proceso\&btnG=Buscar\&hl=es\&as_sdt=2000; consultado 30 de Setiembre, 2010).

FAO. 2010. Food quality assurance and certification. (http:// www.fao.org/ag/ags/subjects/en/agribusiness/foodquality.html; consultado 7 de Agosto, 2010).

Færgemand, J. 2008. The ISO 22000 series: global standards for safe food supply chains. ISO Management Systems, Geneva, Switzerland.
Huss, H. 1994. Assurance of seafood quality. FAO. (http://www. fao.org/DOCREP/003/T1768E/T1768E00.htm\#TOC; consultado 7 de Agosto, 2010).

INTECO. 2006. Sistemas de gestión de la calidad-fundamentos y vocabulario. INTE-ISO 9000: 2005. San José, Costa Rica.

INTECO. 2010. Sistemas de gestión de la calidad. (http://www.inteco.or.cr/esp/index.html; consultado 29 de Abril, 2010).

ISO. 2009. Prerequisite programmes on food safety-Part 1: Food Manufacturing. ISO Management Systems, Switzerland.

Leitón, P. 2010. BAC apoyará a PYMES en eficiencia energética. (http://www.nacion.com/2010-04-23/Economia/NotasSecundarias/Economia2344471.aspx; consultado 28 de Abril, 2010).

Mateos, M., M. Marino, \& A. Castellano. 2009. Diversidad productiva y de estrategias empresarias en las PYMES queseras: políticas a implementar para aumentar su competitividad. (http:// www.inta.gov.ar/baLcarce/info/documentos/econo/DiversidadProductiva.pdf; consultado 30 de Setiembre, 2010).

Mayorga, G. 2010. Banca para el desarrollo prestó en febrero c852 millones a PYMES (http://www.nacion.com/201003-29/Economia/UltimaHora/Economia2319098.aspx; consultado 28 de Abril, 2010).

MINECO, CONACYT, MIFIC, SIC \& MEIC. 2007. Reglamento técnico centroamericano de buenas prácticas de manufactura para industria de alimentos y bebidas procesados. (http: //www.senasa.go.cr/.../union\%20aduanera\%20procesados.pdf, consultado el 28 de Abril, 2010).

Mora, E. 2008. Comunicación personal: curso PIA. CITA. UCR, San Pedro, San José, Costa Rica.

Murillo, M. 2009. Gira de investigación al CITTED en La Perla de San Carlos. Exposición sobre generalidades del CITTED. La Perla, San Carlos.

Programa Estado de la Nación. 2009. Resumen: oportunidades, estabilidad y solvencia económicas. (http://www.estadonacion.or.cr/index.htm; consultado 22 de Abril, 2010). 


\section{APÉNDICE 1}

Entrevista a realizar a los tres productores sobre el estatus de su negocio en términos de inocuidad, productividad y calidad

\begin{tabular}{|c|c|c|c|c|c|c|}
\hline \multicolumn{7}{|c|}{ Entrevista sobre el Sistema de Gestión de la Calidad } \\
\hline \multirow{3}{*}{$\begin{array}{l}\text { Nombre del entrevistador: } \\
\text { Persona a entrevistar: } \\
\text { Fecha: }\end{array}$} & & & & & & \\
\hline & & & & & & \\
\hline & & & & & & \\
\hline \multirow{2}{*}{\multicolumn{7}{|c|}{ Instrucciones }} \\
\hline & & & & & & \\
\hline \multirow{2}{*}{\multicolumn{7}{|c|}{ Coloque un "Si" si el aspecto que está inspeccionando está bien. Si no cumple con lo que se le está pidiendo coloque "No". }} \\
\hline & & & & & & \\
\hline \multicolumn{7}{|l|}{ Entrevista } \\
\hline \multirow{2}{*}{\begin{tabular}{|l|} 
Aspectos a corroborar \\
1. Inocuidad y buenas prácticas o \\
\end{tabular}} & Cumple? No cumple? & Observaciones & Aspectos a corroborar & Cumple? & No cumple? & Observaciones \\
\hline & de manufactura & & 6. Empaque yetiquetado & & & \\
\hline $\begin{array}{l}\text { 1. } 2 \text { Se ha dado entrenamiento en el } \\
\text { lavado de manosy BP M en general? } \\
\text { ¿C ada cuánto se da este } \\
\text { entenamiento? ¿Se llevanregistros? }\end{array}$ & & & $\begin{array}{l}\text { 1.ise cuenta con un procedimiento } \\
\text { que describa el diseño y desarollo de } \\
\text { empaques y etiquetas? }\end{array}$ & & & \\
\hline $\begin{array}{l}\text { 2. ¿C uentan con procedimientos } \\
\text { estandarizados pararealizar la limpiezz } \\
\text { de los equipos e instalaciones?. ¿Se } \\
\text { cuenta con entrenamiento en este } \\
\text { sentido? }\end{array}$ & & & $\begin{array}{l}\text { ¿Seregistran to dos los diseñosy } \\
\text { desarrollos? }\end{array}$ & & & \\
\hline $\begin{array}{l}\text { 3. ¿Se cuenta con un programa de } \\
\text { contol de plagas por escrito? ¿Se ha } \\
\text { dado capacitación? }\end{array}$ & & & $\begin{array}{l}\text { 3. ¿ Seregistran los sro ductos en el } \\
\text { ministerio de salud? }\end{array}$ & & & \\
\hline \begin{tabular}{|l|} 
4. ¿Cuentan con procedimientos \\
estandarizados pararealizar el contol \\
de la inocuidad del agua?. ¿Se cuenta \\
con entrenamiento en este sentido?
\end{tabular} & & & $\begin{array}{l}\text { 4. ¿Se siguen las normas de } \\
\text { etiquetado para todos los } \\
\text { productos? }\end{array}$ & & & \\
\hline \multicolumn{3}{|l|}{ 2. Controlde calidad } & 7. Control de producto terminac & do & & \\
\hline $\begin{array}{l}\text { 1. } 2 \text { Cuentan con un procedimiento } \\
\text { estandarizado para el control de } \\
\text { calidad de las mater ias primas? }\end{array}$ & & & $\begin{array}{l}\text { 1. Se cuenta con un procedimiento } \\
\text { escrito donde se describa el contontol } \\
\text { F FO para el producto terminado? }\end{array}$ & & & \\
\hline 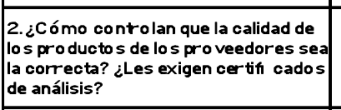 & & & \begin{tabular}{l|} 
2. ¿Se ouenta con un prooedimiento \\
escritt donde se describa el contol \\
de produoto no conforme, desechos \\
producto en curentenay manejo de \\
devoluciones?
\end{tabular} & & & \\
\hline $\begin{array}{l}\text { 3. ¿Se cuenta con un programa de } \\
\text { contol Fo Fo por escrito? ¿Se ha dado } \\
\text { capacitación? }\end{array}$ & & & $\begin{array}{l}\text { 3. ¿Se ha entrenado al personal en } \\
\text { este sentido? }\end{array}$ & & & \\
\hline \multicolumn{3}{|l|}{ 3.Metrología } & 8. Distribución y comercializaci & ión & & \\
\hline $\begin{array}{l}\text { 1. ¿Se ouenta con un manual en donde } \\
\text { se especith que el contol metológico } \\
\text { que se deberealizar en cada equipo? }\end{array}$ & & & \begin{tabular}{|l|} 
1. Tienen deff nido el mercado del \\
producto?
\end{tabular} & & & \\
\hline \begin{tabular}{|l|}
$\begin{array}{l}\text { 2. ¿Se ha dado capacitación en este } \\
\text { tema? }\end{array}$ \\
\end{tabular} & & & $\begin{array}{l}\text { 2. ¿Quué canales de distribución } \\
\text { utilizan? }\end{array}$ & & & \\
\hline \multicolumn{3}{|l|}{ 4. Mantenimiento preventivo } & $\begin{array}{l}\text { 3. ¿Qué cuidados se tóman en la } \\
\text { cadena de distribución? }\end{array}$ & & & \\
\hline $\begin{array}{l}\text { 1. ¿Se cuenta con un procedimiento } \\
\text { general de mantenimiento preventivo? }\end{array}$ & & & \begin{tabular}{|l|} 
4. i Serevisa el producto que está en \\
los puntos de venta?
\end{tabular} & & & \\
\hline $\begin{array}{l}\text { 2. ¿Se cuenta con la descripción del } \\
\text { mantenimiento preventivo que se le } \\
\text { debe dar a cada uno de los sequipos? }\end{array}$ & & & 9. Sevicio al cliente & & & \\
\hline $\begin{array}{l}\text { 3. ¿Se cuenta con un plan de rabajo } \\
\text { para asegurarse de que se cumplan } \\
\text { todas las especifit cacioness de } \\
\text { mantenimiento establecidas? }\end{array}$ & & & $\begin{array}{l}\text { 1. Se lleva un control de los } \\
\text { reclamovo que el cliente gener a? }\end{array}$ & & & \\
\hline $\begin{array}{l}\text { 4. ¿Se cuenta con metas establecidas } \\
\text { semestral o anualmente para que el } \\
\text { mantenimiento correctivo baje? }\end{array}$ & & & $\begin{array}{l}\text { 2. ¿Serealiza una investigación de } \\
\text { cada caso para determinar las } \\
\text { causas del reclamo y asegurar que el } \\
\text { mismo no velva a ocurrir? }\end{array}$ & & & \\
\hline \multicolumn{3}{|l|}{ 5. Control de proceso } & $\begin{array}{l}\text { 3. ¿Se lleva un contol de las entregas } \\
\text { a tiempo? }\end{array}$ & & & \\
\hline 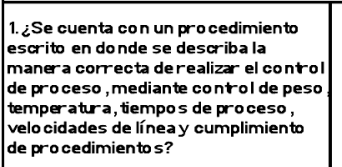 & & & 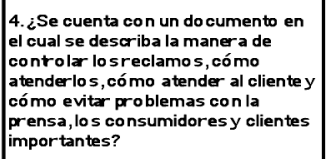 & & & \\
\hline \begin{tabular}{|l|} 
2.2Se cuenta con un procedimiento \\
escrito dondes se describa cómo \\
calcular la et ciencia, efi cacia, \\
desperdicios, etc?
\end{tabular} & & & $\begin{array}{l}\text { 5. ¿Se entrena al personal en el } \\
\text { servicio al cliente? }\end{array}$ & & & \\
\hline & & & & & & \\
\hline \multicolumn{7}{|c|}{ Firma del entevistado: } \\
\hline \multicolumn{3}{|c|}{ Firma del entevistador: } & & & & \\
\hline
\end{tabular}

Fuente: Autora. 


\section{APÉNDICE 2}

Lista de chequeo para determinar el cumplimiento de las buenas prácticas de manufactura en una planta de alimentos

\begin{tabular}{|c|c|c|c|c|c|}
\hline \multicolumn{6}{|c|}{ Lista de chequeo para verificación de la correcta aplicación de las Buenas prácticas en una Agr oindustria } \\
\hline \multirow{2}{*}{\multicolumn{6}{|c|}{ Nombre del inspector: }} \\
\hline & & & & & \\
\hline \multicolumn{6}{|l|}{ Area a inspeccionar: } \\
\hline \multicolumn{6}{|l|}{ Instrucciones } \\
\hline & & & & & \\
\hline \multicolumn{6}{|c|}{ Coloque un "Si" si el aspecto que está inspeccionando está bien. Si no cumple con lo que se le está pidiendo coloque "No". } \\
\hline & & & & & \\
\hline \multicolumn{6}{|l|}{ Hoja de inspección } \\
\hline Aspectos a comoborar & Cumple? No cumple? & & & & \\
\hline \multicolumn{3}{|l|}{ Aspectos a corroborar } & \multicolumn{3}{|l|}{$\begin{array}{l}\text { Aspectos a corroborar } \\
\text { 3. Limpieza y desinfección }\end{array}$} \\
\hline Uñas cortas & & & $\begin{array}{l}\text { Se utilizan detergentesy } \\
\text { desinfectantes para industia } \\
\text { alimentariay conregistro del } \\
\text { ministerio de Salud }\end{array}$ & & \\
\hline Uniforme & & & $\begin{array}{l}\text { Registros de limpieza llenados } \\
\text { correctamentey con la frecuencia } \\
\text { necesaria }\end{array}$ & & \\
\hline Manos limpias & & & $\begin{array}{l}\text { Utensilios en buen estado y } \\
\text { correctos }\end{array}$ & & \\
\hline Sin aretes, maquillaje, perfume & & & $\begin{array}{l}\text { Químicos de limpieza no se } \\
\text { almacenan junto con alimentos }\end{array}$ & & \\
\hline Zapatos cerrados & & & 4. Plagas & & \\
\hline Capacitación en BPM & & & Mapa de trampas & & \\
\hline $\begin{array}{l}\text { Personal enfermo: este personal se } \\
\text { controla para evita contacto en áreas } \\
\text { crítcas del proceso }\end{array}$ & & & Trampas aseguradas al piso & & \\
\hline 2. Instalaciones & & & Ausencia de insectos & & \\
\hline Arededores limpios & & & Fumigaciones al día & & \\
\hline Basureros con tapay rótulo & & & $\begin{array}{l}\begin{array}{l}\text { Productos aprobados por el } \\
\text { Ministerio de Salud }\end{array} \\
\end{array}$ & & \\
\hline Desagües conrejilla & & & Hojas de seguridad de los productos & & \\
\hline Paredes limpias & & & $\begin{array}{l}\text { Si se almacenan los productos, están } \\
\text { aislados delresto? }\end{array}$ & & \\
\hline Cedazo en ventanas & & & \begin{tabular}{|l|l} 
Equipo y utensilios s \\
Fáciles de limpiar
\end{tabular} & & \\
\hline $\begin{array}{l}\text { Singoteras } \\
\text { Rincones sucios }\end{array}$ & & & \begin{tabular}{|l|} 
Fáciles de limpiar \\
$\begin{array}{l}\text { Sin areglos impro visados (cinta } \\
\text { adhesiva) }\end{array}$
\end{tabular} & & \\
\hline $\begin{array}{l}\text { Sistema de ratamiento de desechos } \\
\text { en buenas condiciones }\end{array}$ & & & $\begin{array}{l}\text { No deben tener piezas o tornillos } \\
\text { sueltos }\end{array}$ & & \\
\hline Ausencia de rendijas & & & $\begin{array}{l}\text { No ransfi eren al producto } \\
\text { sustancias tóxicas }\end{array}$ & & \\
\hline B añosy lockers en buen estado & & & $\begin{array}{l}\text { Se evidencia el M antenimiento } \\
\text { preventivo }\end{array}$ & & \\
\hline Unionesredondeadas & & & 5. Control de proceso & & \\
\hline Pisos sinrendijas & & & $\begin{array}{l}\text { Registros del control físico químico } \\
\text { de la po tabilidad del agua }\end{array}$ & & \\
\hline Protectores en las lámparas & & & $\begin{array}{l}\text { Todo saco, bo lsa o caja de producto } \\
\text { contaminado se desecha }\end{array}$ & & \\
\hline Ausencia de go teo o condensación & & & $\begin{array}{l}\text { Conrol del cumplimiento del } \\
\text { diagrama de } 1 \text { ujo (concentraciones, } \\
\text { cantidades correctas) }\end{array}$ & & \\
\hline $\begin{array}{l}\text { Existen lavamanos a la entrada de } \\
\text { cada área separada de la planta }\end{array}$ & & & Control de tiempos & & \\
\hline $\begin{array}{l}\text { Existe jabón, papel to alla o secador } \\
\text { con are calientey alco hol en gel en } \\
\text { cada estación? }\end{array}$ & & & 6. Almacenamiento & & \\
\hline $\begin{array}{l}\text { Existe separación ente tarimasy } \\
\text { paredes de al menos } 5 \mathrm{~cm} \text { y del suelo } \\
20 \mathrm{~cm} ?\end{array}$ & & & $\begin{array}{l}\text { La bodega de producto terminado } \\
\text { está limpiay ordenada }\end{array}$ & & \\
\hline $\begin{array}{l}\text { Todo pro ducto está etiquetado con } \\
\text { fecha de vencimiento y código interno } \\
\text { de control de la empresa }\end{array}$ & & & $\begin{array}{l}\text { La bo dega de mater ia prima está } \\
\text { limpiay ordenada }\end{array}$ & & \\
\hline \multicolumn{5}{|c|}{ Firma de inspector: } & \\
\hline Visto bueno del supervisor de producc: & ión o Calidad:__ & & & & \\
\hline
\end{tabular}

Fuente: Autora. 


\section{APÉNDICE 3}

Plan de mejora propuesto para las microempresas de acuerdo a las debilidades encontradas con las listas de chequeo

\begin{tabular}{|c|c|c|c|c|}
\hline \multirow[b]{2}{*}{ Hallazgo } & \multicolumn{3}{|c|}{ Empresa a la que aplica } & \multirow[b]{2}{*}{ Acción correcti va } \\
\hline & Tostadora & Productora de jugo de Nori & Productora de cajetas & \\
\hline \multicolumn{5}{|l|}{ Pers onal no tiene uñas cortas } \\
\hline Pers on al no utiliza uniforme & & & $x$ & Dis eñar un uniforme para los empleados \\
\hline $\begin{array}{l}\text { No existen facilidades para tener las } \\
\text { manos limpias }\end{array}$ & $\mathrm{x}$ & $x$ & $\mathrm{x}$ & Debe existi $r$ una facilidad para el lavado de manos \\
\hline \multicolumn{5}{|l|}{ El pesronasl utiliza joyas o maquillaje } \\
\hline \multicolumn{5}{|l|}{ El pesronas I no utiliza zapatos cerrados } \\
\hline No ha habido capacitación en BPM & $\mathrm{x}$ & $\mathrm{x}$ & $x$ & Debe darse capacit ación en BPM \\
\hline $\begin{array}{l}\text { No existe una política contra el personal } \\
\text { enfermo }\end{array}$ & $x$ & & $x$ & $\begin{array}{l}\text { Se debe es cribir una políti ca contra el personal enfermo para que no manipule } \\
\text { alimentos directamente }\end{array}$ \\
\hline Los alre de dores no están limpios & & $\mathrm{x}$ & & Alrededores deben estar siempre limpios \\
\hline Los basureros no tienen tapa ni rótulo & $\mathrm{x}$ & $\mathrm{x}$ & $x$ & Basureros deben tener tapa y rótulo \\
\hline Los des agües no tienen rejilla & & & $x$ & Colocar rejilla en desagues \\
\hline Las paredes no están limpias & & & $x$ & Limpiar paredes al menos una vez por semana \\
\hline No existe ced azo en las ventanas & & & $x$ & Colocar cedaz 0 en ventanas \\
\hline \multicolumn{5}{|l|}{ Existen goteras } \\
\hline $\begin{array}{l}\text { Presencia de rincones de difícil acceso } \\
\text { para limpieza }\end{array}$ & & $\mathrm{x}$ & $x$ & No debe haber rinœnes difí ciles de limpiar \\
\hline $\begin{array}{l}\text { No existe un sistema de tratamiento de } \\
\text { desechos en buenas condiciones }\end{array}$ & $x$ & $x$ & & Implementar un sistema de tratamient o de des echos \\
\hline Presencia de rendijas & $\mathrm{x}$ & & $x$ & Cubrir rendijas para prevenir plagas \\
\hline Baños y lock ers en mal estado & & $\mathrm{x}$ & $x$ & Deben estar limpios y en buen estado \\
\hline Uniones no redondead as & $\mathrm{x}$ & & $x$ & Las uniones deben ser redondeadas para evitar crecimiento de plagas \\
\hline Pisos con rendijas & $\mathrm{x}$ & $\mathrm{x}$ & & Deben abrirse læ rendijळ para evitar la entrada de plag ð \\
\hline Aus encia de rl otectores en las lámparas & $\mathrm{x}$ & & & $\begin{array}{l}\text { Colocar protecores en las lámparas para evit ar que se acumulen plas a y que } \\
\text { caigan encima del alimento si sellegaran a quebrar. }\end{array}$ \\
\hline Presencia de goteo o condens ación & $\mathrm{x}$ & $\mathrm{x}$ & $x$ & Arreglar los problemas que provoquen condensación por tuberías con fugas \\
\hline $\begin{array}{l}\text { Aus encia de jabón, papel to alla o } \\
\text { secador con aire caliente y alcohol en } \\
\text { gel en cada estación }\end{array}$ & $\mathrm{x}$ & & $x$ & $\begin{array}{l}\text { Debe existi } r \text { un mantenimiento en cada estación de lavamanos con jabón, alcoho } \\
\text { en } g \text { el y toallas }\end{array}$ \\
\hline $\begin{array}{l}\text { Aus encia de separ ación entre tarimas y } \\
\text { paredes de al menos } 15 \mathrm{~cm} \text { y del suelo } \\
20 \mathrm{~cm}\end{array}$ & $\mathrm{x}$ & $\mathrm{x}$ & & Las tarimas en las bodega deben estarseparadas de la pared y el piso \\
\hline \multicolumn{5}{|l|}{$\begin{array}{l}\text { Los productos no están etiquetados con } \\
\text { fecha de vencimiento y código interno } \\
\text { de control de la empresa }\end{array}$} \\
\hline \multicolumn{5}{|l|}{$\begin{array}{l}\text { No se utilizan detergentes y } \\
\text { desinfectantes para industria alimentaria } \\
\text { y con re gistro del ministerio de Salud }\end{array}$} \\
\hline $\begin{array}{l}\text { Registros de limpieza no se llenan } \\
\text { corr ectamente y con la frecuencia } \\
\text { neces aria }\end{array}$ & $\mathrm{x}$ & & $x$ & Se deben llenar registros de limpieza \\
\hline $\begin{array}{l}\text { Utensilios no es tán en buen estadoy } \\
\text { correctos }\end{array}$ & $x$ & $\mathrm{x}$ & & $\begin{array}{l}\text { Los utensilios de limpieza deben ser los correctos para que la limpieza s ea } \\
\text { correcta }\end{array}$ \\
\hline \multicolumn{5}{|l|}{$\begin{array}{l}\text { Los químicos de limpieza se almacenan } \\
\text { junto con alimentos }\end{array}$} \\
\hline \multicolumn{5}{|l|}{$\begin{array}{l}\text { No existe un mapa de trampas de } \\
\text { I atones }\end{array}$} \\
\hline Trampas no as eguradas al piso & $x$ & $x$ & $x$ & Las trampas de ratones deben est ar aseguradas al piso \\
\hline Presencia de ins ectos & & $\mathrm{x}$ & $x$ & La pres encia de ins ect os implia falta de limpieza y fumiga cón \\
\hline \multicolumn{5}{|l|}{ F umigaciones no están al día } \\
\hline \multicolumn{5}{|l|}{$\begin{array}{l}\text { Productos no aprobados por el } \\
\text { Ministerio de Salud }\end{array}$} \\
\hline $\begin{array}{l}\text { Aus encia de hojas de seguridad de los } \\
\text { productos }\end{array}$ & $\mathrm{x}$ & $x$ & $x$ & Deben existi rhojas de seguridad de los productos \\
\hline $\begin{array}{l}\text { Los productos de fumigación no están } \\
\text { separados del res to de los productos de } \\
\text { la fábrica }\end{array}$ & $\mathrm{x}$ & $\mathrm{x}$ & $x$ & Los productos de fumig ación deben estar separados del res to de los productos \\
\hline
\end{tabular}




\section{APÉNDICE 4}

\section{Plan de mejora propuesto para las microempresas de acuerdo a}

las debilidades encontradas con la encuesta aplicada

\begin{tabular}{|c|c|c|c|c|}
\hline \multirow{2}{*}{ Hallazgo } & \multicolumn{3}{|c|}{ Empresa que no cumple con las preguntas } & \multirow{2}{*}{ Accióncorrecti va } \\
\hline & Tostadora & Productora de jugo de Noni & Productora de cajetas & \\
\hline $\begin{array}{l}\text { ¿Se cuenta con un programa de control de plagas por } \\
\text { escrito? ¿Se ha dado capacitación? }\end{array}$ & $x$ & $x$ & $\mathrm{x}$ & Escribir el documento respecti vo y los registros relacionados \\
\hline $\begin{array}{l}\text { ¿Cuentan con procedimientos estandarizados para } \\
\text { realizar el control de la inocuidad del agua?. ¿Se cuenta } \\
\text { con entrenamiento en este sentido? }\end{array}$ & $\mathrm{x}$ & & $\mathrm{x}$ & Escribir el documento respecti vo y los registros relacionados \\
\hline $\begin{array}{l}\text { ¿Cuentan con un procedimiento estandarizado para el } \\
\text { control de calidad de las materias primas? }\end{array}$ & $x$ & $x$ & $\mathrm{x}$ & Escribir el documento respedi vo y los registros relacionados \\
\hline $\begin{array}{l}\text { ¿Cómo controlan que la calidad de los productos de los } \\
\text { proveedores sea la correcta? ¿Les exigen certificados } \\
\text { de análisis? }\end{array}$ & & $x$ & $x$ & Escribir el documento respecti vo y los registros relacionados \\
\hline $\begin{array}{l}\text { ¿Se cuenta con un programa de control FIF O por } \\
\text { escrito? ¿Se ha dado capacitación? }\end{array}$ & & & & Escribir el documento respecti vo y los registros relacion ados \\
\hline $\begin{array}{l}\text { ¿Se cuenta con un manual en donde se especifique el } \\
\text { control metrológico que se debe realizar en cada } \\
\text { equipo? }\end{array}$ & $x$ & $x$ & $\mathrm{x}$ & Escribir el documento respecti vo y los registros relacion ados \\
\hline $\begin{array}{l}\text { ¿Se cuenta con un procedimiento general de } \\
\text { mantenimiento preventivo? }\end{array}$ & & $x$ & $\mathrm{x}$ & Escribir el documento respecti vo y los registros relacionados \\
\hline $\begin{array}{l}\text { ¿Se cuenta con la descripción del mantenimiento } \\
\text { preventivo que se le debe dar a cada uno de los } \\
\text { equipos? }\end{array}$ & $\mathrm{x}$ & $x$ & $\mathrm{x}$ & Escribir el documento respecti vo y los registros relacionados \\
\hline $\begin{array}{l}\text { ¿Se cuenta con un plan de trabajo para as egurarse de } \\
\text { que se cumplan todas las especificaciones de } \\
\text { mantenimiento establecidas? }\end{array}$ & $x$ & $x$ & $\mathrm{x}$ & Se deben revisar registros para evitar mantenimiento correcti vo \\
\hline $\begin{array}{l}\text { ¿Se cuenta con metas establecidas semestral } 0 \\
\text { anualmente para que el mantenimiento correctivo baje? }\end{array}$ & $\mathrm{x}$ & $\mathrm{x}$ & $\mathrm{x}$ & Revisar metas de manera periódica \\
\hline $\begin{array}{l}\text { ¿Se cuenta con un procedimiento escrito en donde se } \\
\text { describa la manera correcta de realizar el control de } \\
\text { proceso, mediante control de peso, temper atura, } \\
\text { tiempos de proceso, velocidades de líneay } \\
\text { cumplimiento de proce dimientos? }\end{array}$ & $x$ & $x$ & & Escribir el documento respecti vo y los registros relacion ados \\
\hline $\begin{array}{l}\text { ¿Se cuenta con un procedimiento escrito donde se } \\
\text { describa cómo calcular la eficiencia, eficacia, } \\
\text { desperdicios, etc? }\end{array}$ & $x$ & $x$ & $\mathrm{x}$ & Escribir el documento respecti vo y los registros relacion ados \\
\hline $\begin{array}{l}\text { ¿Se cuenta con un procedimiento que describa el } \\
\text { diseño y desarrollo de empaques y etiquetas? }\end{array}$ & $x$ & $x$ & $\mathrm{x}$ & Escribir el documento respecti vo y los registros relacionados \\
\hline ¿Se registran todos los diseños y desarrollos? & $x$ & $x$ & $\mathrm{x}$ & Debe existi $r$ regis tro de todos los empaques y eti quetas que se han uti liz \\
\hline ¿Se registran los productos en el ministerio de salud? & $\mathrm{x}$ & $\mathrm{x}$ & $\mathrm{x}$ & El ministerio de salud emite un registro que sirve de res paldo para lafàbrica \\
\hline $\begin{array}{l}\text { \& ¿Se siguen las normas de etiquetado para todos los } \\
\text { productos? }\end{array}$ & $x$ & $x$ & $x$ & Seguir las normas del MEIC \\
\hline $\begin{array}{l}\text { ¿Se cuenta con un procedimiento escrito donde se } \\
\text { describa el control FIF } 0 \text { para el producto ter min ado? }\end{array}$ & & $x$ & $x$ & Escribir el documento respecti vo y los registros relacion ados \\
\hline $\begin{array}{l}\text { ¿Se cuenta con un procedimiento escrito donde se } \\
\text { describa el control de producto no conforme, desechos, } \\
\text { producto en cuarentenay manejo de devoluciones? }\end{array}$ & & $x$ & $x$ & Escribir el documento respecti vo y los registros relacion ados \\
\hline ¿Se ha entrenado al personal en este sentido? & $\mathrm{x}$ & $x$ & $\mathrm{x}$ & Revisar metas de manera periódica \\
\hline \multicolumn{5}{|l|}{ ¿Tienen definido el merca do del producto? } \\
\hline \multicolumn{5}{|l|}{ ¿Qué canales de distribución utilizan? } \\
\hline \multicolumn{5}{|l|}{ ¿Qué cuidados se toman en la cadena de distribución? } \\
\hline $\begin{array}{l}\text { ¿ Se revis a el producto que está en los puntos de } \\
\text { venta? }\end{array}$ & $\mathrm{x}$ & $x$ & $\mathrm{x}$ & Se debe empezar a revisar los productos en los puntos de venta \\
\hline $\begin{array}{l}\text { ¿Se lleva un control de los reclamos que el cliente } \\
\text { genera? }\end{array}$ & $x$ & & $x$ & $\begin{array}{l}\text { Debe llevarse el registro de los reclamos, son parte muy import ante de la } \\
\text { immegen de la empresa }\end{array}$ \\
\hline $\begin{array}{l}\text { ¿Se realiza una investigación de cada caso par a } \\
\text { determinar las caus as del re clamo y as egurar que el } \\
\text { mismo no vuelva a ocurrir? }\end{array}$ & $x$ & $x$ & $x$ & $\begin{array}{l}\text { Es muy importante realizar la investi gación de coda redamo porque de ahi } \\
\text { salen muchas acciones correcti vas que ayudan a mejorar }\end{array}$ \\
\hline ¿Se lleva un control de las entregas a tiempo? & $\mathrm{x}$ & $x$ & $\mathrm{x}$ & Es importante para uti lizarlo para evaluar la calidad de los proveedores \\
\hline
\end{tabular}

NOTA: Los aspectos incluidos en la lista de chequeo no se incluyeron en este plan de mejora por ser los mismos. 